\title{
Electrical And Computer Engineering Technology Curriculum From \\ The System Design's Perspective
}

\author{
Omer Farook, Chandra R. Sekhar, Jai P. Agrawal, \\ Essaid Bouktache, Ashfaq Ahmed \\ Purdue University Calumet, Hammond, IN 46323
}

\author{
Mohamed Zainulabeddin \\ Electronics Corporation of India Limited, ECIL Post, Hyderabad - 500 062, India
}

\begin{abstract}
Traditionally Electrical and Computer Engineering Technology (ECET) curriculum start with two courses in digital switching theory, one addresses combinational logic and the other sequential logic. Both of these courses typically are discrete components centric (TTL or CMOS). The paper provides a totally fresh look at Electrical Engineering Technology curriculum from the perspective of System Design. It examines the subject areas that prepares the student to pursue the discipline of System Design from at least four different perspectives; via: 1) PLD/FPGA centric system design, 2) Microcontroller based Embedded System Design, 3) PC based Network-oriented Distributed System Design and 4) DSP based Real-time Processing based System Design.

The paper discusses a set of courses in the area of hardware, software, firmware, networking and DSP, which provides a road map in the form of curriculum that utilizes the same tools which industry is employing. This approach to curriculum bridges the gap that exists between the classroom practices and industrial practices. This unified approach would deliver students with career-bound knowledge essential for the industry.
\end{abstract}

\section{Introduction}

In the Electrical and Computer Engineering Technology (ECET) department of Purdue University Calumet (PUC), the faculty by choice have taken upon themselves to make the changes, the essence of which is reflected in this paper. After a lot of deliberation the curriculum committee agreed to make the changes to the curriculum that essentially, prepares the student to function in the market place as the System Designer. It was realized that the ECET curriculum should impart to the graduating student enough exposure to pursue the discipline of System Design from at least four different perspectives; via: 1) PLD/FPGA centric system design, 2) Microcontroller based Embedded System Design, 3) PC based Network-oriented Distributed System Design 
and 4) DSP based Real-time Processing based System Design. The Industrial Advisory committee was fully supportive in this process and provided constructive suggestions in this regard. It was with this objective that curriculum overhaul was done and sequences of two courses at the minimum were slated in each of the four areas. Thus a total of eight courses were designed and are being offered over a four-year time span, spread among eight semesters of bachelor's degree program in ECET.

\section{PLD/FPGA Centric System Design}

Historically ECET programs across the nation have always started with a two-course sequence in Digital Logic Design. The first course covers the Combinational Logic Design and the second course covers the Sequential Logic Design. Both of these courses typically were discrete components centric (TTL or CMOS) ${ }^{[1,2]}$. Our department keeping the same Lecture-hours, Lab-hours and Credit hours has redefined these courses so that they are now PLD/FPGA centric ${ }^{[3,4]}$. This change was warranted, in the light of industrial practices and in terms of maturation of this technology and tools.

Programmable Logic Devices have become extremely popular. PLDs can provide design security and copy protection. They enable a design to be changed simply and cheaply without making expensive and time consuming PCB changes as earlier random logic designs required. Finally, PLDs are a lot more "fun" to work with than random TTL or CMOS logic (Student centric outlook!). Thus with these two course sequence the students become proficient in carrying their system design with PLDs and FPGAs. The general consensus of the system design community is that: the designs should begin in HDL, followed by some amount of simulation to verify the credibility of design. Static timing analysis should follow, to discover the worst-case paths in the design ${ }^{[5]}$.

The text ${ }^{[3]}$ introduces Complex PLDs [CPLDs] early in the teaching sequence. Student learns the new paradigm shift in the digital design early on with their very first course. The first Digital Design course [ECET 109, Lecture 2: Lab. 2: Credit 3] covers the following topics:

- Basic Principles of Digital Systems, Logic functions and gates, Boolean Algebra and Combinational Logic.

- Introduction to PLDs and MAX+PLUS II software by Altera Corporation.

- $\quad$ Student learn to program PLDs using, MAX+PLUS II.

- Design simple combinational circuits using schematic capture.

- Use Very High Speed Integrated Circuit [VHSIC] VHSIC Hardware description Language [VHDL] declarations, architecture and concurrent signal assignments to enter simple combinational circuits.

- Create circuit symbols from schematics or VHDL designs for PLDs.

- Assign device and pin numbers to schematic or VHD designs and compile them to programming of Altera MAX 7000S and FLEX10K type devices.

- Various combinational Logic functions and arithmetic circuits are primarily pursued using PLDs and VHDL programming. 
The second Digital Design course in this sequence [ECET 159, 3:3:4] begins with, introduction to sequential Logic. Then the following topics are covered:

- Design applications using NAND/NOR Latches, Gated Latches, D, JK and T Flip-Flops.

- Programmable Logic Architecture of devices [MAX700S and FLEX10K] is explored.

- Counters and Shift Register design is implemented using MAX+PLUS II Graphic Design File or in VHDL.

- The course culminates with the "classic" (state table) method of the State Machine Design. This leads to the determination of the Boolean equations of the State Machine. The next step is to translate Boolean equation of the State Machine into graphical Design File. Finally write VHDL code to implement the State Machine Design.

\section{Microcontroller based Embedded System Design}

Most of the ECET programs have at least one introductory course in either Microprocessor or Microcontroller. Typically such a course is taught in Assembly language. Our department also followed very much the same route. Over a period that spans over two decades, we engaged in objective self-analysis and took a number of corrective actions. This evolutionary process took us from 8085, 8088 microprocessors to 8051 Microcontroller and then to currently used Microchip ${ }^{[8]}$ PIC 16F84 Microcontroller $^{[6]}$. During this long haul objective analysis found a number of flaws to traditional teaching. Most of the semester is utilized or (wasted) in learning of the Assembly Language. The remainder of the semester is devoted to the architecture of the Microprocessor /Microcontroller. For most of the simple systems' design Microcontroller are inherently better suited. For the beginning student the Microcontroller route provides all the functionality in a neat package. Our choice has been PIC 16F84. We have departed from Assembly language. All the software design for the PIC 16F84 is done in C Language ${ }^{[6,7]}$.

The ECET department at PUC, has replaced a single (Class 3, Lab 3, Credit 4) course (ECET 209, 3: 3: 4) with two courses (ECET209 and ECET 309). Each of these courses have, (Class 3, Lab 3, Credit 4) pattern. This has essentially doubled the Class and Lab time devoted to Embedded System Design area.

Thus to summarize the changes: a) Moved from Assembly language to C language, $b$ ) Selected to teach Microchip PIC Microcontroller(s), c) Doubled the lecture time and lab time; have made a marked improvement in students ability to carry on with confidence the design work in the area of Embedded System Design. This change has been reflected through the number of projects completed in Senior Design. Many of these projects have significantly improved in sophistication and complexity. 


\section{PC Based Network-oriented Distributed System Design}

System Design with an embedded Microprocessor was the course that gradually evolved into System Design with PC components over a period of 15 years or so. The course (ECET 456, 3:3:4) Computer Hardware System Design, currently utilizes the PC technology components that are readily available in so many different vintages. These components are used in the System Design. The course covers the architectural details of the Intel's Pentium processor(s) ${ }^{[9]}$, deals with memory design (ideal as well as protected modes), cover the interfacing with the Operating System (Windows). Different standard buses (ISA, PCI and USB) are utilized for port interfacing and data and control signals for the system. The course utilizes $\mathrm{C}^{++}$Language for all the Software System Design ${ }^{[10]}$ needs.

The follow up second course could take a number of different routes: 1) This course could be pursed (Software System Design) totally in Windows GUI environment (ECET 455). Window's GUI Software System Design is an area that is in high demand in the market place, but virtually shunned by the academic community. The platform for the software design is Borland $\mathrm{C}^{++}$Builder. This approach provides the student to program in entirely GUI, Object Oriented Programming environment. 2) This course requires a background in Networking, and by adding the component of socket programming and connectivity, the system truly becomes distributed. ECET department has offered this course (ECET 499) experimentally, during the past few semesters.

The impact of the above sequence of courses could be measured by the fact that $75 \%$ of the senior design projects utilize the core knowledge gained. PC based System Design, provides a convenient mechanism to design any customize system, regardless of end usage. Provides the student the knowledge base for Hardware, Software Integration.

\section{DSP Based Real-time Processing based System Design}

Digital Signal Processing clearly prepares the student to handle real time processing of data and signal in a Variety of applications almost in every field. The ECET department at PUC currently has a two-course sequence in this regard (ECET 384 and ECET 492). The text is unique in bringing the subject matter to the grasp of students and is appropriate for undergraduate level ${ }^{[11]}$. A supplemental text is also used for reference purposes and its soft copy is free of charge available on $\mathrm{NET}^{[12]}$.

The first course covers the mathematical foundation of Discrete Signal and System representation. The course content of ECET 384 is as follows:

- Analog-To-Digital and Digital-To-Analog Conversion

- Digital Signals

- Difference Equations and Filtering

- Convolution and Filtering

- Z Transforms 
- Fourier Transforms and Filter Shape

- Digital signal Spectra

The second course deals with, all the classical DSP techniques. Again all the DSP Chip Programming is to be performed via C Language. The course content of ECET 492 is as follows:

- Finite Impulse Response Filters

- Infinite Impulse Response Filters

- Discrete Fourier Transform and Fast Fourier Transform Processing

- Hardware for DSP

- Programming DSPs

- Signal Processing

- Image Processing

- Wavelets

The ECET department feels that even though the current two-course sequence in the area of DSP, provides the students with a good understanding of the methodology and principles, it falls short of making them productive as DSP System Designers. One-way to address this is, by offering a graduate level DSP Applications course.

\section{Software Design}

Over the course of time, in our discipline (ECET) Software has been gaining foothold as the medium of functional implementation of the design. This phenomenon provides the System Design with flexibility and cut cost at the same time. Much of today's Engineering Technologist effectiveness could be attributed to Software Design Skills. ECET department with this realization has implemented a two-course sequence in $\mathrm{C}^{++}$ Language (ECET 110, 2:2:3 and ECET 210 2:2:3). Students take these two courses in their very first year, which provides them with sufficient software background for a life long career.

\section{Conclusion}

The curriculum changes that are implemented by the Electrical and Computer Engineering Technology Department at Purdue University Calumet as outlined here have integrated the areas of disciplines (PLD/FPGA centric system design, Microcontroller based Embedded System Design, PC based Network-oriented Distributed System Design and DSP based Real-time Processing based System Design) that make our student industry ready. A two-course sequence approach in different subject areas has provided the pedagogical framework for the students to master the subject area before moving on to next area of emphasis. The approach outlined in this paper still provides a common thread of continuity throughout: that of, System Design. Another thread that runs through out the curriculum is: Software Design. Post graduation surveys of our students have pointed to the fact that: "Software Design" as an attribute has made them invaluable for their employers. 


\section{Bibliography}

[1] Computer Logic Design, M. Morris Manu, ISBN: 0-13-165472-1

Prentice Hall. Copyright: 1972

[2] Digital Systems, Principles and Applications. Ronald Tocci

ISBN: 0-13-304262-6, Prentice Hall. Copyright:1995

[3] Digital Design with CPLD Applications and VHDL, Robert Dueck

ISBN: 07668-1160-3. Publisher: Delmar,Thomson Learning. Copyright: 2001

[4] Digital Electronic with VHDL, William Kleitz, Tompkins,

ISBN: 0-13-110080-7. Publisher: Prentice Hall. Copyright: 2004

[5] Programmable devices take on the vestiges of regular logic, Craig Matsumoto, EE Times October 31, 2000

[6] Design with PIC Microcontrollers, John B. Peatman, ISBN: 0-13-759259-0

Publisher: Prentice Hall, Copyright: 1998

[7] Embedded C programming and the Microchip PIC,, Barnet, COX \& O'Cull.

ISBN:140183748-4. Publisher: Delmar,Thomson Learning. Copyright: 2004

[8] Microchip corporation: http://www.microchip.com

[9] Intel Microprocessors 8086/8088, 80186/80188, 80286, 80386, 80486 Pentium, Pentium Pro Processor, Pentium II, Pentium III, and Pentium IV: Architecture, Programming, and Interfacing, 6/E, Barry B. Brey, ISBN: 0-13-060714-2

Publisher: Prentice Hall

[10] Applied PC Interfacing, Graphics and Interrupts, W. Buchanan, ISBN: 0-201-8778-7

publisher: Addison Wesley Longman,

[11] Fundamentals of Digital Signal Processing, Joyce Van de Vegte,

ISBN: 0-13-016077-6,

Publisher: Prentice Hall

[12] The Scientist and Engineer's Guide to Digital Signal Processing, by Steven W. Smith, California Technical Publishing, ISBN 0-9660176-3-3 (1997)

http://www.dspguide.com

Proceedings of the 2004 American Society for Engineering Education Annual Conference \& Exposition

Copyright (C) 2004, American Society for Engineering Education 


\section{Biographical Information}

OMER FAROOK is a member of the faculty of the Electrical and Computer Engineering Technology Department at Purdue University Calumet. Professor Farook received the Diploma of Licentiate in Mechanical Engineering and BSME in 1970and 1972 respectively. He further received BSEE and MSEE in 1978 and 1983 respectively from Illinois Institute of Technology. Professor Farook's current interests are in the areas of Embedded System Design, Hardware-Software Interfacing, Digital Communication, Networking, Distributed Systems, $\mathrm{C}^{++}$and Java Languages. He is a member of ASEE and senior member of IEEE.

CHANDRA R. SEKHAR is a member of the faculty of the Electrical and Computer Engineering Technology at Purdue University Calumet. Professor Sekhar earned a Bachelor's Degree in Chemistry from the University of Madras (India), a Diploma in Instrumentation from Madras Institute of Technology and Master's Degree in Electrical Engineering from University of Pennsylvania. Professor Sekhar's primary teaching and research focus is in the areas of Biomedical and Process Control Instrumentation and Clinical Engineering.

JAI AGRAWAL is a Professor with joint assignment in Electrical and Computer Engineering Technology and Electrical \& Computer Engineering. He received his PH.D. in Electrical Engineering from University of Illinois, Chicago, in 1991, M.S. and B.S. also in Electrical Engineering from I.I.T. Kanpur, India in 1970 and 1968 respectively. Professor Agrawal has worked recently for two years in optical networking industry in the Silicon Valley in California. Professor Agrawal is the Founder Advisor to Agni Networks Inc., San Jose, California. His expertise includes optical networking at Physical and Data link layers, optical and WDM interface, SONET and Gigabit Ethernet and analog electronic systems. He is the author of a Textbook in Power Electronics, published by Prentice-Hall. His professional career is equally divided in academia and industry. He has authored several research papers in IEEE journals and conferences.

ESSAID BOUKTACHE is a member of the faculty of the Electrical and Computer Engineering Technology Department at Purdue University Calumet. Dr. Bouktache received his MS and Ph. D in Electrical Engineering from the Ohio State University in 1980 and 1985, respectively. His research and teaching interests include Digital Signal Processing, Computer Networks, and Digital Communications. Professor Bouktache has been with Purdue since 1992 and is a member of IEEE and ASEE. He has several publications to his credit.

ASHFAQ AHMED is Professor of Electrical and Computer Engineering Technology Department at Purdue University Calumet. Professor Ahmed received his Bachelors of Science degree in Electrical Engineering from the University of Karachi in 1973 and Master of Applied Science degree in 1978 from University of Waterloo. He is the author of a Textbook in Power Electronics, published by Prentice-Hall. $\mathrm{He}$ is a registered professional engineer in the state of Indiana. He is senior member of IEEE. Professor Ahmed's current interests are in the areas of Embedded System Design, $\mathrm{C}^{++}$and Networking,

MOHAMED ZAINULABEDDIN is Deputy General Manager with Electronics Corporation of India Limited, Hyderabad. In this capacity he is responsible for defining Corporate Executive Learning Process. During his tenure at ECIL, he has integrated emerging technologies in the corporate culture and has created an environment of life long learning for the employees. Mr. Zainulabeddin received the Diploma of Licentiate in Electrical Engineering in 1966. He received his BSEE and MTEC in 1975 and 1987 respectively from Jawaher-Lal Nehru Technological University. From $1966-73$, he served as lecturer in Govt. Polytechnique, Hyderabad. He is currently over seeing the development of Need Base Management Development Programs for officers and Senior Executives of ECIL.

Proceedings of the 2004 American Society for Engineering Education Annual Conference \& Exposition Copyright $(\underset{2}{2004}$, American Society for Engineering Education 